\title{
Optimal power-to-mass ratios when predicting flat and hill-climbing time-trial cycling
}

Accepted: 17 March 2006/Published online: 10 May 2006

(C) Springer-Verlag 2006

\begin{abstract}
The purpose of this article was to establish whether previously reported oxygen-to-mass ratios, used to predict flat and hill-climbing cycling performance, extend to similar power-to-mass ratios incorporating other, often quick and convenient measures of power output recorded in the laboratory [maximum aerobic power ( $\left.W_{\text {MAP }}\right)$, power output at ventilatory threshold $\left(W_{\mathrm{VT}}\right)$ and average power output $\left(W_{\mathrm{AVG}}\right)$ maintained during a $1 \mathrm{~h}$ performance test]. A proportional allometric model was used to predict the optimal power-to-mass ratios associated with cycling speeds during flat and hill-climbing cycling. The optimal models predicting flat time-trial cycling speeds were found to be $\left(W_{\mathrm{MAP}} m^{-0.48}\right)^{0.54},\left(W_{\mathrm{VT}} m^{-0.48}\right)^{0.46}$ and $\left(W_{\mathrm{AVG}} m^{-0.34}\right)^{0.58}$ that explained $69.3,59.1$ and $96.3 \%$ of the variance in cycling speeds, respectively. Cross-validation results suggest that, in conjunction with body mass, $W_{\text {MAP }}$ can provide an accurate and independent prediction of time-trial cycling, explaining $94.6 \%$ of the variance in cycling speeds with the standard deviation about the regression line, $s=0.686 \mathrm{~km} \mathrm{~h}^{-1}$. Based on these models, there is evidence to support that previously reported $\dot{V} \mathrm{O}_{2}$-tomass ratios associated with flat cycling speed extend to other laboratory-recorded measures of power output (i.e. $W^{-0.32}$ ). However, the power-function exponents
\end{abstract}

A. M. Nevill $(\bowtie) \cdot$ S. A. Jobson

Research Institute of Healthcare Sciences, Simon Jobson,

School of Sport, Performing Arts and Leisure,

University of Wolverhampton, Gorway Road,

Walsall WS1 3BD West Midlands, England

E-mail: a.m.nevill@wlv.ac.uk

Tel.: + 44-1902-322838

Fax: +44-1902-322898

R. C. R. Davison

Department of Sport and Exercise Science,

University of Portsmouth, Portsmouth, Hampshire, England

A. E. Jeukendrup

School of Sport and Exercise Sciences,

The University of Birmingham,

Edgbaston, Birmingham, West Midlands, England
$(0.54,0.46$ and 0.58$)$ would appear to conflict with the assumption that the cyclists' speeds should be proportional to the cube root (0.33) of power demand/ expended, a finding that could be explained by other confounding variables such as bicycle geometry, tractional resistance and/or the presence of a tailwind. The models predicting 6 and $12 \%$ hill-climbing cycling speeds were found to be proportional to $\left(W_{\mathrm{MAP}} m^{-0.91}\right)^{0.66}$, revealing a mass exponent, 0.91, that also supports previous research.

Keywords Power supply and demand - Cycling speed Maximal aerobic power $\left(W_{\mathrm{MAP}}\right) \cdot$ Power at ventilatory threshold $\left(W_{\mathrm{VT}}\right) \cdot$ Average power output $\left(W_{\mathrm{AVG}}\right)$

\section{Introduction}

There is still some confusion as to what are the most appropriate power-to-mass ratios associated with athletic performance. Nevill et al. (1992) described two quite distinct types of power-to-mass ratio when scaling physiological variables such as maximum oxygen uptake, peak and mean power $(Y)$, for individuals of different body masses $(m)$. The first, known as the 'power-function' ratio, $\mathrm{Ym}^{-k}$ (based on the allometric model, $Y=a$ $m^{k}$, where $a$ is the scaling constant and $k$ the body-mass scaling exponent), renders the physiological variable independent of body mass. Based on their empirically derived power-function models, Nevill et al. (1992) proposed that the best method of scaling maximal oxygen uptake $\left(\dot{V} \mathrm{O}_{2 \max }\right)$, peak and mean power output $(W)$ required these variables to be divided by $m^{2 / 3}$. However, recently a number of studies investigating the relationship between metabolic rate (either basal or maximal) have questioned this simplistic approach and reported mass exponents greater than $2 / 3$ or 0.67 (see Darveau et al. 2002). Indeed, Heil (2005) recently confirmed, using a reanalysis of data reported by Coyle et al. (1991), that the average power output ( $\left.W_{\mathrm{AvG}}\right)$ maintained during a $1 \mathrm{~h}$ performance test of elite and non-elite cyclists was 
proportional to body mass, $m^{0.684}$, similar to but a little greater than that expected for measures of $\dot{V} \mathrm{O}_{2 \max }$ assuming subjects are geometrically similar to each other (an assumption that is also questionable, see Nevill et al. 2004). The main purpose of these power-function ratios is to facilitate valid comparison of physiological variables (usually in epidemiological type studies) between groups (or individuals) of different body sizes.

The second 'scaling' ratio described by Nevill et al. (1992) was derived to optimally predict running performance, recorded as run speed $\left(\mathrm{m} \mathrm{s}^{-1}\right)$, using the allometric model, speed $\left(\mathrm{m} \mathrm{s}^{-1}\right)=a_{2}(Y)^{k_{1}}(m)^{k_{2}}$. It is important to recognise that this second ratio is not a true 'scaled' index. Indeed when predicting running performance, such indices are inclined to over-adjust the participants' physiological performance variables for body size, i.e. the correlation between the optimal ratio, maximum oxygen uptake $\left(\mathrm{ml} \mathrm{kg}^{-1} \mathrm{~min}^{-1}\right)$ and body mass $(\mathrm{kg})$ was found to be negative for men and women (Nevill et al. 1992). To distinguish between these two ratios, Nevill et al. (1992) refers to the former as representing the participants' physiological capacity, i.e. their ability to utilise oxygen or record power maximally, independent of body size, and the latter as representing the subjects' performance capacity or ability to run which is highly dependent on body size. These two terms are equivalent to the power supply and power demand of cycling described by Heil (2005). In this article, we wish to investigate the second power demand or performance capacity ratio, i.e. the most appropriate power-to-mass ratio to predict cycling performance (recorded as an average speed) over flat and hilly terrain, using power output recorded in a laboratory.

Swain (1994) was probably the first to estimate the mass exponent, 0.32 , associated with oxygen consumption when investigating the energy cost of cycling over flat ground at sub-maximal speeds $(20,15$ and $10 \mathrm{mph})$. As a result, a number of authors have adopted the ratio, $\dot{V} \mathrm{O}_{2}(m)^{-0.32}$ as the optimal oxygen-to-mass ratio associated with sub-maximal and maximal cycling performances. However, only recently were Nevill et al. (2005) able to confirm a similar association exists when predicting time-trial cycling performances using $\dot{V} \mathrm{O}_{2 \max }$ and body mass as the predictor variables incorporated in the allometric model described above (Nevill et al. 1992). The authors found the optimal $\dot{V} \mathrm{O}_{2 \max }$-to-mass ratio to predict average time-trial cycling speed was $\left(\dot{V} \mathrm{O}_{2 \max }(m)^{-0.32}\right)^{0.41}$. Not only did this power-function ratio confirm that the anticipated optimal oxygen-tomass ratio was given by $\dot{V} \mathrm{O}_{2 \max }(m)^{-0.32}$ but also that the association between cycling speed and oxygen cost is curvilinear (being constrained by air resistance), thought to be proportional to the cube root of the energy expended, $\left(V \mathrm{O}_{2 \max }(m)^{-0.32}\right)^{0.41}$.

A number of authors (Padilla et al. 1999; Lucia et al. 2004) have adopted the same ratio structure when using maximal power output $\left(W_{\max }\right)$ as a predictor of level/flat cycling performance (i.e. $\dot{V} \mathrm{O}_{2}$ was simply replaced by $W_{\max }$ ), dividing $W_{\max }$ by $m^{0.32}$. However, the assumption that these ratios can be applied/extended to measures of power output $(W)$ does not appear to have been established/confirmed when predicting time-trial cycling speed performance as outlined by Nevill et al. (1992) above.

There has been little research on the optimal oxygenor power-to-mass ratios associated with hill-climbing cycling performance. Swain (1994) was probably the first to establish that a body-mass exponent of 0.79 was associated with the oxygen cost of uphill cycling $(10 \%$ gradient at $11.3 \mathrm{~km} \mathrm{~h}^{-1}$ ). Heil (1998) also explored the oxygen cost of uphill cycling (gradients of 1.7, 3.5, 5.2 and $7 \%$ ) at a constant speed of $3.46\left(\mathrm{~m} \mathrm{~s}^{-1}\right)=12.46$ $\left(\mathrm{km} \mathrm{h}^{-1}\right)$. The body-mass exponents associated with the oxygen cost of uphill cycling did not differ significantly across the four gradients, found to be 0.89 [given by the slopes of the regression analysis of $\log \left(\dot{V} \mathrm{O}_{2}\right)$ on $\left.\log (m)\right]$. Several authors have successfully used these mass exponents to predict hill-climb time-trial performance (Heil et al. 2001) and to stratify professional cyclists according to their expertise (i.e. TT-, uphill-specialist, etc.) (Padilla et al. 1999). However, as far as we are aware, no research has directly confirmed/validated these ratios and the curvilinear associations between uphill cycling speed and both mass and power output as predictors, see Nevill et al. (2005).

Given that power output is thought to be a better predictor of cycling performance than oxygen consumption (Bishop et al. 1998; Lucia et al. 2004), the purpose of the present article is to establish whether the oxygen-to-mass ratios described above do indeed apply/ extend to other measures of power output recorded in the laboratory [maximum aerobic power output ( $\left.W_{\mathrm{MAP}}\right)$, power output at ventilatory threshold $\left(W_{\mathrm{VT}}\right)$ and average power $\left(W_{\mathrm{AVG}}\right)$ maintained during a $1 \mathrm{~h}$ performance test] when predicting flat and hill-climbing time-trial cycling performance. As the primary resistance to movement whilst cycling is proportional to air resistance, we shall adopt similar proportional allometric or power-function models adopted by Nevill et al. (1992, 2005). This will not only assess the optimal power-tomass ratios associated with flat and hill-climbing cycling performances, but also accommodate the anticipated proportional curvilinear association between time-trial cycling speeds and energy expenditure.

\section{Methods}

The methods used during studies 1, 2 and 3 have previously been described in detail (see Nevill et al. 2005; Coyle et al. 1991; Davison et al. 2000, respectively). As such, the following sections provide only a summary.

\section{Study 1}

\section{Participants}

Twenty-three male cyclists were recruited from local cycling clubs to participate in this investigation 
(participant characteristics presented in Table 1). Participants were competitive but non-elite cyclists.

\section{Testing schedule}

Each participant visited the laboratory for a $2 \mathrm{~h}$ test session to determine participant characteristics. On a separate occasion, participants performed a competitive 25 mile $(40.23 \mathrm{~km})$ road time-trial. The laboratory session and the time-trial event were completed in a random order separated by no more than 10 days during the months of May, June and July.

\section{Laboratory testing}

Participants completed a progressive, incremental exercise test to exhaustion on a Kingcycle air-braked cycle ergometer (Kingcycle Ltd, High Wycombe, Buckinghamshire, UK). This system, which has previously been described in full (Palmer et al. 1996), allows the participant to exercise on their own bicycle against a resistance comparable to that of riding on the road. This system has been shown to be both valid and reliable during 20 , $40 \mathrm{~km}$ (Palmer et al. 1996) and max-test protocols (Keen et al. 1991).

Following standardised calibration procedures (Palmer et al. 1996), participants carried out a warm-up at a self-selected intensity for $10 \mathrm{~min}$. Immediately following this, the maximal test was initiated at a workload of 150-200 W. Thereafter, workload increased at a ramp rate of $20 \mathrm{~W} \mathrm{~min}^{-1}(1 \mathrm{~W}$ every $3 \mathrm{~s})$. The test was terminated when the participant could no longer maintain the specified workload, despite strong verbal encouragement, given by the same investigator during all maximal trials. Maximal aerobic power ( $\left.W_{\mathrm{MAP}}\right)$, not to be confused with maximal oxygen uptake $\left(\dot{V} \mathrm{O}_{2 \max }\right)$, was calculated as the highest average power output $(W)$ over a $60 \mathrm{~s}$ period using the Kingcycle software.

For the duration of the maximal test protocol, respiratory gasses were recorded on a breath-by-breath basis using an Oxycon Pro automated gas analysis system (Erich Jaeger GmBH, Hoechberg, Germany).

Power output at ventilatory threshold $\left(W_{\mathrm{VT}}\right)$ was calculated as the power output $(W)$ corresponding with a systematic increase in the ventilatory equivalent of oxygen $\left(V \mathrm{E} / \dot{V} \mathrm{O}_{2}\right)$ without a concomitant increase in the ventilatory equivalent of carbon dioxide $\left(V \mathrm{E} / V \mathrm{CO}_{2}\right)$ (Amann et al. 2004).

\section{Road time-trials}

Participants competed in one of five competitive 25 mile time-trial events, each being carried out on a flat 'outand-back' course and according to the regulations of the Road Time Trials Council (RTTC 2004). Note that the five time-trial events were conducted on separate days during the months of May, June and July, using four individual courses (two events used the same course). Based on Ordnance Survey maps (Ordnance Survey, Southampton, UK), the average elevations for the five courses were $105( \pm 7), 55( \pm 3), 95( \pm 11), 50( \pm 4)$ and $105( \pm 7) \mathrm{m}$ above sea level (note that courses one and five were the same). Based upon data recorded at a local weather station, wind speed was $0,0.8( \pm 0.37), 16.1$ $( \pm 0.75), 10.7( \pm 0.98)$ and $0 \mathrm{~km} \mathrm{~h}^{-1}$ for the five TT events. Due to the nature of the out-and-back TT courses used, average wind direction was predominantly a crosswind with respect to the overall course direction. Mean temperature, barometric pressure and humidity for each of the five time-trial events are presented in Table 2.

Following a warm-up of self-selected duration and intensity, all participants used geared road bicycles fitted with 'aero' bars to complete the race distance as quickly as possible. No drafting was permitted. For the completed 25 mile time-trial, total time (min) and average speed $\left(\mathrm{km} \mathrm{h}^{-1}\right)$ were calculated.

\section{Study 2}

\section{Participants}

Fifteen male competitive USCF category 1 or 2 cyclists were recruited for this study (participant characteristics presented in Table 1). Subjects whose recent best time for a $40 \mathrm{~km}$ time-trial was faster than $56 \mathrm{~min}$ were identified as group 1 ('elite-national class'). Subjects with slower performances were placed in group 2 ('good-state class').

\section{Laboratory testing}

Each participant completed two separate laboratory sessions. During the first session participant characteristics were recorded. This involved the determination of subject training/competition history and maximal oxy-

Table 1 Physical characteristics of participants from Studies 1, 2 and 3

\begin{tabular}{|c|c|c|c|c|c|c|c|c|c|}
\hline & $N$ & Age (years) & $\pm \mathrm{SD}$ & Body mass $(\mathrm{kg})$ & $\pm \mathrm{SD}$ & $\mathrm{W}_{\text {MAP }}$ & $\pm \mathrm{SD}$ & $\dot{\mathrm{V}} \mathrm{O}_{2 \max }\left(1 \min ^{-1}\right)$ & $\pm \mathrm{SD}$ \\
\hline Study 1 & 23 & 41.3 & 10.9 & 78.0 & 9.4 & 336 & 45.9 & 4.29 & 0.55 \\
\hline Study 2 & 15 & 23.1 & 3.5 & 72.0 & 6.6 & - & - & 4.97 & 0.41 \\
\hline Study 3 & 8 & 26.5 & 6.9 & 73.3 & 5.2 & 357 & 19.6 & $4.43^{\mathrm{a}}$ & 0.24 \\
\hline
\end{tabular}

Means \pm SD ( = standard deviation $)$

${ }^{a}$ Estimated using a predictive regression equation (see Keen et al. 1991) 
Table 2 Environmental data for the five time-trial events used in Study 1

\begin{tabular}{|c|c|c|c|c|c|c|c|}
\hline & $N$ & Temperature $\left({ }^{\circ} \mathrm{C}\right)$ & $\pm \mathrm{SD}$ & Pressure (mbar) & $\pm \mathrm{SD}$ & Humidity (\%) & $\pm \mathrm{SD}$ \\
\hline Race 1 & 4 & 20.8 & 0.25 & 1,028 & 0.5 & 50.0 & 0 \\
\hline Race 2 & 6 & 14.5 & 0.62 & 1,025 & 0 & 75.7 & 2.3 \\
\hline Race 3 & 4 & 16.9 & 1.49 & 1,009 & 0 & 61.8 & 5.6 \\
\hline Race 4 & 6 & 21.4 & 1.31 & 1,027 & 0.2 & 45.9 & 5.7 \\
\hline Race 5 & 3 & 19.9 & 0.20 & 1,005 & 0 & 52.5 & 0.9 \\
\hline Mean & & 18.5 & 3.02 & 1,021 & 9.3 & 58.0 & 12.5 \\
\hline
\end{tabular}

Means \pm SD $(=$ standard deviation $)$

gen consumption. $\dot{V} \mathrm{O}_{2 \max }$ was determined during a continuous, incremental laboratory cycle test lasting between 8 and $10 \mathrm{~min}$, using a Monark cycle ergometer (model 819) equipped with a racing seat, drop handlebars and pedals for cleated shoes. During the second laboratory session, participants performed a $1 \mathrm{~h}$ laboratory-based performance test on the Monark ergometer from which average power output $\left(W_{\mathrm{AvG}}\right)$ was recorded. In attempting to maximise the amount of work performed in $1 \mathrm{~h}$, subjects were allowed to vary both flywheel resistance and pedal cadence.

\section{Road time-trials}

The recent best time for each subject during a flat $40 \mathrm{~km}$ road time-trial was recorded.

Study 3 (6 and 12\% hill climbs)

\section{Participants}

Eight male competitive cyclists were recruited for this investigation (participant characteristics presented in Table 1). Although participants were familiar with maximal laboratory testing, all cyclists underwent an extensive habituation period to become confident with treadmill riding.

\section{Laboratory testing}

Determination of maximal aerobic power output. Each participant completed an incremental exercise test lasting 10-12 min, conducted using a Kingcycle test rig (described above). The test was initiated at a power output dependent upon the cyclist's body mass or ability. The workload was increased at a rate of $20 \mathrm{~W} \mathrm{~min}-1$ until volitional exhaustion. Maximum aerobic power ( $\left.W_{\mathrm{MAP}}\right)$ was calculated as the highest average minute power output.

Hill-climb performance tests. Participants completed two $6 \mathrm{~km}$ climbs at $6 \%$ gradient and two $1 \mathrm{~km}$ climbs at $12 \%$ gradient on a treadmill (Woodway XELG70, Weil am Rhein, Germany). During all tests cyclists were asked to complete the distance as fast as possible. The treadmill was initially set at a manageable speed determined from the habituation rides; the cyclists could then request an increase or decrease in speed as desired.

\section{Statistical methods}

In order to establish the most appropriate power-tomass ratio to best reflect cycling speed, the following power-function model (Nevill et al. 1992, 2005) was used to explore the optimal relationship between cycle speed, power output $(W)$ (either $W_{\mathrm{MAP}}, W_{\mathrm{VT}}$ or $W_{\mathrm{AVG}}$ ) and body mass $(m)$,

Cycle speed $\left(\mathrm{km} \mathrm{h}^{-1}\right)=a(W)^{k_{1}}(m)^{k_{2}} \varepsilon$,

where $a$ is a constant and $k_{1}$ and $k_{2}$ the exponents likely to provide the best predictor of cycling speed and $\varepsilon$ the multiplicative error ratio. The model can be linearised with a $\log$ transformation, and multiple linear regression can be used to estimate the unknown parameters $a, k_{1}$ and $k_{2}$. The log-transformed model becomes

$\begin{aligned} \log _{\mathrm{e}}(\text { speed })= & \log _{\mathrm{e}}(a)+k_{1} \log _{\mathrm{e}}(W)+k_{2} \log _{\mathrm{e}}(m) \\ & +\log _{\mathrm{e}}(\varepsilon) .\end{aligned}$

Note that the parameter $a$ can be allowed to vary between groups (e.g. between the five time-trial events), thus conducting a form of analysis of covariance (ANCOVA).

In order to assess the validity of the above power-tomass models for flat time-trial cycling performance, we cross-validated the model for cycling speed from Study 1. This was achieved by predicting the speeds of the cyclists in Study 2 using the model from Study 1 but adopting the power $\left(W_{\mathrm{AVG}}\right)$ and body-mass data as predictor variables from Study 2 (Coyle's study). The success of the cross-validation was assessed by comparing the actual speeds from Study 2 with the predicted speeds.

\section{Results}

Study 1

The ANCOVA identified no difference in cycling speeds between the five time-trial events (i.e. there was no difference in the constant $a$ for the five time-trial events, $P>0.05)$ together with no difference in the $W_{\text {MAP }}$ 
(W $\min ^{-1}$ ) and body mass $m(\mathrm{~kg})$ covariate slope parameters. Hence, the optimal power-function model relating average cycle speed $\left(\mathrm{km} \mathrm{h}^{-1}\right)$ to $W_{\text {MAP }}$ ( $\mathrm{W} \min ^{-1}$ ) and body mass, $m(\mathrm{~kg})$, was found to be common for all five time-trial events, given by,

Cycle speed $\left(\mathrm{km} \mathrm{h}^{-1}\right)=5.1\left(W_{\mathrm{MAP}}\right)^{0.54}(m)^{-0.26}$,

with $\quad \mathrm{R}$-sq $=69.3 \% \quad\left(k_{1}=0.541, \quad \mathrm{SEE}= \pm 0.08 \quad\right.$ and $k_{2}=-0.257, \mathrm{SEE}= \pm 0.09$ ) and the error ratio (the standard deviation of residuals about the fitted log-linear regression model, Eq. 1), $s=0.0457$ or $4.68 \%$, having taken antilogs. The model predicts the mean cycle speed for the time-trials was $38.6 \mathrm{~km} \mathrm{~h}^{-1}$ (predicted for an average participant whose $W_{\mathrm{MAP}}=333 \mathrm{~W} \mathrm{~min} \mathrm{~m}^{-1}$ and body mass $=77.5 \mathrm{~kg}$ ). Note that the above cyclingspeed model for relatively flat time-trial cycling can be rearranged and expressed as a power-to-mass ratio within a curvilinear power function as follows:

$$
\begin{aligned}
& \text { Cycle speed } \approx\left(W_{\mathrm{MAP}}\right)^{0.54}(m)^{-0.26} \\
&=\left(W_{\mathrm{MAP}}\right)^{0.54}\left(m^{-0.48}\right)^{0.54}=\left(W_{\mathrm{MAP}} m^{-0.48}\right)^{0.54}, \\
& \text { since }(m)^{-0.26}=\left(m^{-0.48}\right)^{0.54} .
\end{aligned}
$$

The $95 \%$ confidence interval (CI) for the rearranged/ rescaled mass exponent -0.48 was from -0.15 to -0.81 $(P<0.05)$.

We also explored, using the same power-function model, whether the power output at ventilatory threshold $\left(W_{\mathrm{VT}}\right)$ and body mass might be better predictors of average cycle speed $\left(\mathrm{km} \mathrm{h}^{-1}\right)$. The resulting power-tomass ratio was similar, proportional to $\left(W_{\mathrm{VT}}\right)^{0.46}$ $(m)^{-0.22}=\left(W_{\mathrm{VT}} m^{-0.48}\right)^{0.46}$ but the explained variance was less $\mathrm{R}$-sq $=59.1 \%\left(k_{1}=0.462, \mathrm{SEE}= \pm 0.09\right.$ and $k_{2}=-0.217, \mathrm{SEE}= \pm 0.106$ ), with an error ratio (the standard deviation of residuals about the fitted log-linear regression model, Eq. 1), $s=0.053$ or $5.4 \%$, having taken antilogs. The $95 \%$ confidence interval (CI) for the rearranged/rescaled mass exponent -0.48 was from -0.02 to $-0.94(P<0.05)$.

\section{Study 2}

The average cycling speed $\left(\mathrm{km} \mathrm{h}^{-1}\right)$ of the elite and nonelite cyclists recorded during a $40 \mathrm{~km}$ time-trial was predicted using the average power output $\left(W_{\mathrm{AvG}}\right)$ recorded during a $1 \mathrm{~h}$ test on a stationary cycle ergometer and body mass $(\mathrm{m})$ as predictor variables. The ANCOVA found a significant difference between groups $(P<0.001)$ but no difference in the $W_{\mathrm{MAP}}\left(\mathrm{W} \mathrm{min}^{-1}\right)$ and body mass $m(\mathrm{~kg})$ covariate slope parameters. The optimal allometric model is given by

Cycle speed $($ elite $)=3.46\left(W_{\text {AVG }}\right)^{0.58}(m)^{-0.20}$,

Cycle speed $($ non-elite $)=3.29\left(W_{\mathrm{AVG}}\right)^{0.58}(\mathrm{~m})^{-0.20}$,

with a combined R-sq $=96.3 \% \quad\left(k_{1}=0.58\right.$, $\mathrm{SEE}= \pm 0.08$ and $\left.k_{2}=-0.20, \mathrm{SEE}= \pm 0.07\right)$ and the error ratio (the standard deviation of residuals about the fitted log-linear regression model, Eq. 1), $s=0.014$ or $1.4 \%$, having taken antilogs. A simple rearrangement of the model identified the optimal power-to-mass ratio as proportional to $\left(W_{\mathrm{AVG}}\right)^{0.58}$ $(m)^{-0.20}=\left(W_{\mathrm{AVG}}\right)^{0.58}\left(m^{-0.34}\right)^{0.58}=\left(W_{\mathrm{AVG}} m^{-0.34}\right)^{0.58}$, since $(m)^{-0.20}=\left(m^{-0.34}\right)^{0.58}$, i.e. the mass-denominator exponent was $-0.34(95 \%$ CI from -0.11 to -57 , $P<0.05)$.

The results from the cross-validation process found that the predicted speed (using the model from Study 1, Eq. 2) explained $94.6 \%$ of the variance in the actual speeds of the cyclists from Study 2 with a standard deviation about the regression line of $s=0.686 \mathrm{~km} \mathrm{~h}^{-1}$. The difference in the quality of the cyclists from Study 1 and Study 2 still remained, however, with the mean ( \pm SD) actual and predicted cycling speeds $\left(\mathrm{km} \mathrm{h}^{-1}\right)$ of the cyclists in Study 2 being 42.77 $( \pm 2.7)$ versus $38.5( \pm 1.4)$, respectively $(P<0.01)$. The actual and predicted cycling speeds from the cross-validation assessment can be seen in Fig. 1 .

Study 3 (6 and 12\% hill climbs)

When we pooled both sets of data, the ANCOVA found a significant difference in average cycle speeds between the two simulated hill climbs (6 vs $12 \%$ gradient, $P<0.001)$ but no difference in the $W_{\mathrm{MAP}}\left(\mathrm{W} \mathrm{min}^{-1}\right)$ and body mass $m(\mathrm{~kg})$ covariate slope parameters $(P=0.75$ and $P=0.77$, respectively). Hence, the optimal power-function models relating average cycle speeds $\left(\mathrm{km} \mathrm{h}^{-1}\right)$ at 6 and $12 \%$ gradients to $W_{\text {MAP }}$ (W $\min ^{-1}$ ) and body mass, $m(\mathrm{~kg})$, were found to be

Cycle speed $6 \%\left(\mathrm{~km} \mathrm{~h}^{-1}\right)=5.92\left(W_{\mathrm{MAP}}\right)^{0.66}(\mathrm{~m})^{-0.60}$, Cycle speed $12 \%\left(\mathrm{~km} \mathrm{~h}^{-1}\right)=3.84\left(W_{\mathrm{MAP}}\right)^{0.66}(\mathrm{~m})^{-0.60}$,

with a combined R-sq $=98.3 \% \quad\left(k_{1}=0.66\right.$, $\mathrm{SEE}= \pm 0.16$ and $\left.k_{2}=-0.60, \mathrm{SEE}= \pm 0.13\right)$ and the error ratio (the standard deviation of residuals about the

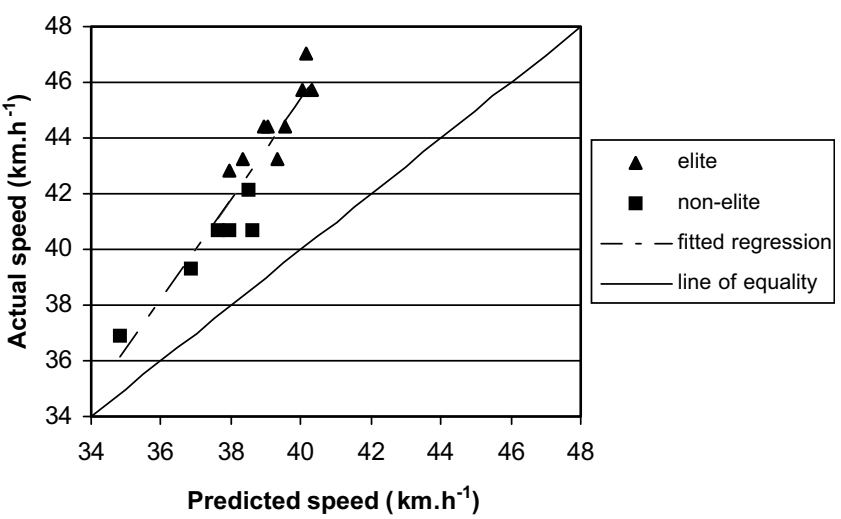

Fig. 1 The actual versus predicted speeds (based on the model from Study 1) of the cyclists from Study 2, together with the fitted regression line and the line of equality 
fitted log-linear regression model, Eq. 1), $s=0.033$ or $3.3 \%$, having taken antilogs. The common power-tomass ratio for 6 and $12 \%$ hill climbing can be expressed as

$$
\begin{aligned}
& \text { Cycle speed } \approx\left(W_{\mathrm{MAP}}\right)^{0.66}(m)^{-0.60} \\
&=\left(W_{\mathrm{MAP}}\right)^{0.66}\left(\mathrm{~m}^{-0.91}\right)^{0.66}=\left(W_{\mathrm{MAP}} m^{-0.91}\right)^{0.66}, \\
& \text { since }(m)^{-0.60}=\left(m^{-0.91}\right)^{0.66} .
\end{aligned}
$$

The $95 \%$ confidence interval $(\mathrm{CI})$ for the rearranged/ rescaled mass exponent -0.91 was from -0.53 to -1.29 $(P<0.01)$.

\section{Discussion}

The results from Study 1 identified the optimal models to predict time-trial cycling speed on relatively flat terrains as being either $\left(W_{\mathrm{MAP}} m^{-0.48}\right)^{0.54}$ or $\left(W_{\mathrm{VT}}\right.$ $\left.m^{-0.48}\right)^{0.46}$ depending on which measure of power output we incorporated into the power-function models. The model incorporating $W_{\mathrm{MAP}}$ explained nearly $70 \%$ of the variance in time-trial cycling performance, $10 \%$ more than the model incorporating the power output at ventilatory threshold $\left(W_{\mathrm{VT}}\right)$ and nearly $30 \%$ more than the model incorporating $\dot{V} \mathrm{O}_{2 \max }$ and body mass previously reported by Nevill et al. (2005). These findings, together with the relative ease with which $W_{\text {MAP }}$ can be assessed, support the utility of power output and, in particular, maximum aerobic power ( $\left.W_{\mathrm{MAP}}\right)$ as a predictor of cycling performance compared with oxygen consumption (Bishop et al. 1998; Lucia et al. 2004).

The results from Study 2 using data originally published by Coyle et al. (1991) identified that the optimal power-to-mass ratio to predict average cycling speed $\left(\mathrm{km} \mathrm{h}^{-1}\right)$ was $\left(W_{\text {AvG }} m^{-0.34}\right)^{0.58}$. The model explained $\mathrm{R}$-sq $=96.3 \%$ of the variance in cycling speed, considerably better than using average $1 \mathrm{~h}$ power $\left(W_{\mathrm{AvG}}\right)$ alone, $\mathrm{R}$-sq $=78.1 \%(r=-0.88)$ originally reported by Coyle et al. (1991).

The models adopting average $1 \mathrm{~h}$ power $\left(W_{\mathrm{AvG}}\right)$ from Study 2 explained more of the variance in cycling speed than $W_{\text {MAP }}$ and $W_{\text {VT }}$ used in Study 1 . However, this is unsurprising given that both the $1 \mathrm{~h}$ performance test and the time-trial field tests are based upon similar performances and hence similar physiological parameters. Furthermore, Study 2 utilises 'best times', likely to be performed under more optimal conditions, which might also help to explain why the power-to-mass ratio from study 2 was able to explain more of the variance in cycling speeds. The advantages of using the $W_{\text {MAP }}$ and $W_{\text {VT }}$ protocols are that they are quick, convenient and provide a strong independent prediction of time-trial cycling performance. Indeed when we cross-validated the power-to-mass model for cycling speed from Study 1 incorporating $W_{\text {MAP }}$ and body mass as predictors (Eq. 2), the model explained $94.6 \%$ of the variance in the actual speeds of the cyclists from Study 2 with impressive accuracy/precision, with the unexplained variation or standard deviation about the fitted regression line being $s=0.686 \mathrm{~km} \mathrm{~h}^{-1}$.

Note that if we assume the power supply is proportional to $m^{0.667}$ as reported in Heil (2005) and replace $W_{\text {AVG }}$ by $m^{0.677}$ in the power function in Study 2, we obtain cycling speed (equivalent to the distance covered in $1 \mathrm{~h}, D_{\mathrm{HR}}$, reported by Heil 2005) proportional to

$$
\begin{aligned}
\text { Cycle speed }\left(\mathrm{km} \mathrm{h}^{-1}\right) & \propto\left(W_{\mathrm{AVG}} m^{-0.34}\right)^{0.58} \\
& =\left(m^{0.667} m^{-0.34}\right)^{0.58}=\left(m^{0.327}\right)^{0.58} \\
& =m^{0.190}
\end{aligned}
$$

very similar to the $m^{0.174}$ reported by Heil (2005). This confirms that bigger/heavier cyclists should perform better (faster) when cycling on relatively flat terrains. We suggest the optimal power-to-mass ratio, e.g. ( $W_{\mathrm{AVG}}$ $\left.m^{-0.34}\right)^{0.58}$, should be referred to as the "performance capacity ratio' (Nevill et al. 1992), whereas the mass exponent, e.g. 0.190, should be referred to as the 'performance capacity mass exponent'.

The above mass exponents $-0.48,-0.48$ and -0.34 (for $W_{\mathrm{MAP}}, W_{\mathrm{VT}}$ and $W_{\mathrm{AVG}}$, respectively) associated with these power-to-mass ratios would appear to be a little greater than the mass exponents, 0.32 , reported by Swain (1994) and Nevill et al. (2005) when assessing the oxygen cost of sub-maximal $(10,15$ and $20 \mathrm{mph})$ and maximal TT cycling performance (approximating maximum steady-state or threshold speeds), respectively. However, the confidence intervals would appear to encompass the 0.32 mass exponent previously reported. Hence, the above mass exponents do not preclude the 0.32 mass exponent previously reported when investigating the $\dot{V} \mathrm{O}_{2}$-to-mass ratios associated with flat cycling speed (i.e. $W^{-0.32}$ ).

The power-function models describing time-trial cycling performance on relatively flat terrains were also able to confirm the anticipated curvilinear association between cycling speed and energy expenditure (Pugh 1974). On level ground, the power demand of cycling is thought to be proportional to the cube of the cyclists' speed (Olds et al. 1995; Heil 2005). Consequently, the speed of a cyclist should also be proportional to the cube root (0.33) of the power expended. However, based on the power-function exponents from Study 1 and Study 2 $[0.54 \quad(\mathrm{SEE}=0.08), \quad 0.46 \quad(\mathrm{SEE}=0.09) \quad$ and 0.58 $(\mathrm{SEE}=0.08)]$, two of the three power-function exponents preclude this theoretical parameter $(0.33)$, i.e. based on $\pm 2 \times$ SEE. This suggests that although the empirically derived exponents confirm the curvilinear association anticipated by Pugh (1974), they conflict with the theoretical parameter 0.33 . However, the theoretical parameter 0.33 is based on the assumption that the cyclists are performing in windless conditions, where the cyclists' ground speed ' $s$ ' is equal to the air speed ' $v$ '. The power expended against aerodynamic resistance, normally expressed as $P=k_{\mathrm{a}} A v^{2} s$, can be simplified under still-wind conditions as $P=k_{\mathrm{a}} A v^{3}$, where ' $k_{\mathrm{a}}$ ' is the form drag coefficient and ' $A$ ' the frontal surface area (Di 
Prampero et al. 1979). However, in the presence of a tailwind $(t),{ }^{\prime} v$ ' is less than ' $s$ ' and hence the power required to maintain a given speed will either become less, given by $P=k_{\mathrm{a}} A(s-t)^{2} s$, or the speed of a cyclist will increase at a rate greater than that implied by the cuberoot relationship 0.33 , possibly approaching the 0.5 value reported in the present article.

Whilst a tailwind may have been present during those time-trials reported by Study 2, the predominant crosswind observed during Study 1 would, in this instance, appear to preclude this explanation. Clearly, a tailwind is not the only explanation that may influence the proposed curvilinear association between time-trial cycling speed and power demand. For example bicycle geometry and tractional resistance (variables not available in the present studies) are other confounding variables that have been shown to affect these associations (Heil 1998, 2001).

As anticipated, the body-mass component of the power-to-mass ratios predicting hill-climbing cycling performances (Study 3) was considerably greater. The power function for 6 and $12 \%$ hill climbing was proportional to $\left(W_{\text {MAP }}(m)^{-0.91}\right)^{0.66}$. The body-mass exponent, 0.91 , is a little greater than that, 0.79 , reported by Swain (1994) when estimating the contribution of the body mass associated with oxygen cost of uphill cycling $\left(10 \%\right.$ gradient at $\left.11.3 \mathrm{~km} \mathrm{~h}^{-1}\right)$, but agrees closely with the mass exponent 0.89 reported by Heil (1998). As with the present study, Heil (1998) also identified no significant difference in body-mass exponents associated with the oxygen cost of uphill cycling, for all gradients ranging between $1.7,3.5,5.2$ and $7 \%$ at a constant speed of $12.46\left(\mathrm{~km} \mathrm{~h}^{-1}\right)$.

Interestingly, if we assume the power supply of cyclists is proportional to $m^{0.667}$ as suggested by Heil (2005) and replace $W_{\text {MAP }}$ by $m^{0.677}$ in the above powerfunction model for hill climbing, we obtain cycling speed (equivalent to the distance covered in $1 \mathrm{~h}, D_{\mathrm{HR}}$, reported by Heil 2005) proportional to

$$
\begin{aligned}
\operatorname{Cycle~speed}\left(\mathrm{km} \mathrm{h}^{-1}\right) & \propto\left(W_{\mathrm{MAP}} m^{-0.91}\right)^{0.66} \\
& =\left(m^{0.667} m^{-0.91}\right)^{0.66}=\left(m^{-0.243}\right)^{0.66} \\
& =m^{-0.160}
\end{aligned}
$$

confirming the negative effect that body mass will have on hill-climbing cycling performance, not dissimilar to the $m^{-0.223}$ reported by Heil (1998).

The power-function models describing hill-climbing performance also identified a curvilinear association between cycling speed and energy expenditure. The fitted model was found to be proportional to ( $W_{\text {MAP }}$ $\left.m^{-0.91}\right)^{0.66}$ for 6 and $12 \%$ gradients. The power-function exponent $(0.66$; SEE $=0.16)$ is greater than those observed when predicting time-trial cycling performance on flat terrain. However, these estimates will reflect the force due to gravity as the dominant force/resistance to hill-climbing motion rather than the negligible wind resistance, given that the adopted protocol in Study 3 was a simulated hill climb performed in the laboratory on a treadmill. Although some research has already validated the power-to-mass ratios in hill-climbing cycling performance (Heil et al. 2001), further research is required to ascertain whether these curvilinear powerfunction exponents are valid when predicting hillclimbing cycling in a 'field' setting.

In conclusion, the results of the present study support the use of laboratory-measured power output (either $W_{\mathrm{MAP}}, W_{\mathrm{VT}}$ or $\left.W_{\mathrm{AVG}}\right)$ when predicting flat and hillclimbing cycling performance. The results suggest that in conjunction with body mass, $W_{\mathrm{VT}}$, and, in particular, $W_{\text {MAP }}$ will provide a better, more accurate prediction of the energy demand of cycling than $\dot{V} \mathrm{O}_{2 \max }$. Although $W_{\mathrm{AvG}}$ did explain $96.3 \%$ of the variance in time-trial cycling speed, this is unsurprising given that the $1 \mathrm{~h}$ laboratory performance relies upon similar performance and hence similar physiological parameters that govern the time-trial field tests. The advantages of using the $W_{\mathrm{MAP}}$ and $W_{\mathrm{VT}}$ protocols are that they are quick, convenient and provide a strong, independent and accurate prediction of time-trial cycling performance. This was evidenced when we cross-validated the powerto-mass ratio model from Study 1 (Eq. 2), used to predict the speeds of the cyclists in Study 2. The predicted speeds explained $94.6 \%$ of the variance in actual speeds from Study 2, with a standard deviation of $s=0.686 \mathrm{~km} \mathrm{~h}^{-1}$. Based on the fitted allometric models from Studies 1 and 2, there is support for the assumption that previously reported $\dot{V} \mathrm{O}_{2}$-to-mass ratios $\left(\dot{V} \mathrm{O}_{2} m^{-0.32}\right)$ associated with flat cycling performance extend to other laboratory-recorded measures of power output. However, the power-function exponents $[0.54 \quad(\mathrm{SEE}=0.08), \quad 0.46 \quad(\mathrm{SEE}=0.09) \quad$ and 0.58 $(\mathrm{SEE}=0.08)]$ were unable to confirm the assumption that the speed of a cyclist performing on level ground is proportional to approximately the cube root $(0.33)$ of the energy demand/expended. The empirically derived power-function exponents appear to be closer to the square root $(0.5)$ of predicted power demand, a finding that might be explained if the cyclists had performed in the presence of a tailwind. The body-mass exponents associated with 6 and $12 \%$ hill climbing, found to be 0.91 , also support previous research.

\section{References}

Amann M, Subudhi A, Foster C (2004) Influence of testing protocol on ventilatory thresholds and cycling performance. Med Sci Sports Exerc 36:613-622

Bishop D, Jenkins DG, Mackinnon LT (1998) The relationship between plasma lactate parameters, W-peak and 1-h cycling performance in women. Med Sci Sports Exerc 30:1270-1275

Coyle EF, Feltner ME, Kautz SA, Hamilton MT, Montain SJ, Baylor AM, Abraham LD, Petrek GW (1991) Physiological and biomechanical factors associated with elite endurance cycling performance. Med Sci Sports Exerc 23:93-107

Darveau CA, Suarez RK, Andrews RD, Hochachka PW (2002) Allometric cascade as a unifying principle of body mass effects on metabolism. Nature 417:166-170 
Davison RCR, Swan D, Coleman D, Bird S (2000) Correlates of simulated hill climb cycling performance. J Sports Sci 18:105-110

Di Prampero PE, Cortili G, Mognoni P, Saibene F (1979) Equation of motion of a cyclist. J Appl Physiol 47:201-206

Heil DP (1998) Scaling of submaximal oxygen uptake with body mass and combined mass during uphill treadmill bicycling. $\mathbf{J}$ Appl Physiol 85:1376-1383

Heil DP (2001) Body mass scaling of projected frontal area in competitive cyclists. Eur J Appl Physiol 85:358-366

Heil DP (2005) Body size as a determinant of the 1-h cycling record at sea level and altitude. Eur J Appl Physiol 93:547-554

Heil DP, Murphy OF, Mattingly AR, Higginson BK (2001) Prediction of uphill time-trial bicycling performance in humans with a scaling-derived protocol. Eur J Appl Physiol 85:374-382

Keen PS, Passfield L, Hale T (1991) Indirect determination of VO2max using a sports-specific (cycling) ergometry system. J Sports Sci 9:420

Lucia A, Hoyos J, Perez M, Santalla A, Earnest CP, Chicharro JL (2004) Which laboratory variable is related with time trial performance time in the Tour de France? Br J Sports Med 38:636-640

Nevill AM, Ramsbottom R, Williams C (1992) Scaling physiological measurements for individuals of different body size. Eur J Appl Physiol Occup Physiol 65:110-117
Nevill AM, Stewart AD, Olds T, Holder RL (2004) Are adult physiques geometrically similar? The dangers of allometric scaling using body mass power laws. Am J Phys Anthropol 124:177-182

Nevill AM, Jobson SA, Palmer GS, Olds TS (2005) Scaling maximum oxygen uptake to predict cycling time-trial performance in the field: a non-linear approach. Eur J Appl Physiol Occup Physiol 94:705-710

Olds TS, Norton KI, Lowe ELA, Olive S, Reay F, Ly S (1995) Modeling road-cycling performance. J Appl Physiol 78:15961611

Padilla S, Mujika I, Cuesta G, Goiriena JJ (1999) Level ground and uphill cycling ability in professional road cycling. Med Sci Sports Exerc 31:878-885

Palmer GS, Dennis SC, Noakes TD, Hawley JA (1996) Assessment of the reproducibility of performance testing on an air-braked cycle ergometer. Int J Sports Med 17:293-298

Pugh LGCE (1974) The relation of oxygen intake and speed in competition cycling and comparative observations on the bicycle ergometer. J Physiol Lond 241:795-808

RTTC (2004) Handbook. Geerings, Ashford

Swain DP (1994) The influence of body-mass in endurance bicycling. Med Sci Sports Exerc 26:58-63 